\title{
La transparencia en la política española: análisis de su influencia y presencia en medios sociales
}

Influence and presence of the concept of transparency in the Spanish social-media political debate

\section{Ana María Morales García, Sara Martínez Cardama, Fátima García López, Mercedes CARIDAD SEBASTIÁN}

Universidad Carlos III de Madrid, Calle Madrid 128, 28903, Getafe; amorales@bib.uc3m.es, smarti1@bib.uc3m.es, fatimag@bib.uc3m.es, mercedes@bib.uc3m.es

\begin{abstract}
Resumen
Se analiza la influencia real del concepto de trans-parencia en la actual política española en el período pre y post electoral (20 de diciembre de 2015). Para ello, se evaluó su presencia en los debates difundidos a través de los medios sociales de los principales partidos políticos (Partido Popular, Partido Socialista Obrero Español, Podemos y Ciudadanos). Los resultados permiten conocer la importancia de la transparencia en el discurso político en medios sociales y cómo se aborda su implementación en un momento de incertidumbre política.
\end{abstract}

Palabras clave: Transparencia. Partidos políticos. España. Medios Sociales. Partido Popular. Partido Socialista Obrero Español. Ciudadanos. Podemos.

\section{Transparencia: marco legal e índices de percepción de la corrupción}

El concepto de transparencia legislativa se inicia en el Siglo XVIII en Suecia, con la aparición de la primera Ley de Transparencia en 1766. Sin embargo, la concepción actual de transparencia deviene del ámbito anglosajón, particularmente de Estados Unidos con la aparición de Ley de Libertad de Información (FOIA) en 1966 (Gastón y Naser, 2012).

Resulta paradójico como países con una gran tradición en materia de acceso a la información pública, tienen ya instaurada en la sociedad la cultura de la transparencia y no precisan de un marco jurídico tan garantista. Esta idea se constata con los datos del Global Right to Information Rating (RTI Rating) que evalúan el marco legal de cada país en materia de acceso a la información. El estudio se basa en la aplicación de 61 indicadores en materia de acceso a la información (Derecho de acceso; Alcance de la ley; Procedimientos de solicitud; Excepciones y rechazos; Apelaciones; Protección y sanciones)

\begin{abstract}
The real influence of the concept of transparency in Spanish politics is analyzed. The analysis covered both pre-election and post-election periods of the 20th December 2015 national Spanish elections. In particular, social media usage of the main political parties (Partido Popular, Partido Socialista Obrero Español, Podemos and Ciudadanos) was monitored and collected. The results show the importance of transparency in the political discourse in Social media and its implementation in a time of political uncertainty.
\end{abstract}

Keywords: . Transparency. Political parties. Spain. Social media. Partido Popular. Partido Socialista Obrero Español. Ciudadanos. Podemos.

Según este ranking, existen 102 países en el mundo que cuentan con leyes de transparencia que obligan a los gobiernos y entidades públicas a hacer públicos sus datos. De estas 102 leyes, solo un $12,7 \%$ aparecieron antes de 1990 , mientras que un $69 \%$ son posteriores al año 2000.

En la siguiente figura pueden observarse las primeras y últimas posiciones del Global Right to Information Rating, teniendo en cuenta que el valor óptimo de este ranking es de 150 puntos.

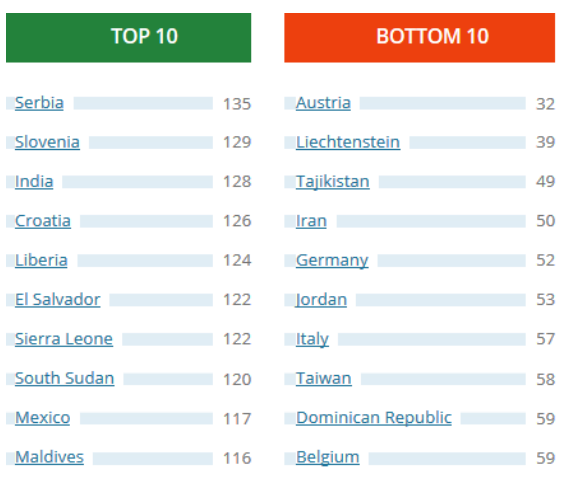

Figura 1. Country Rating Results (Centre for Law and Democracy, 2016) 
Tal como muestran los datos, se observa que países con menores niveles de desarrollo (como India, Liberia o El Salvador) tienen leyes de transparencia de mejor calidad que otros con mayores índices de desarrollo (Austria, Alemania o Bélgica) (Figura 1). Como se puede observar, este ranking está basado en aspectos que no tienen en cuenta la ejecución de las leyes sino su contenido y desarrollo.

Por otra parte, este ranking indica que la reciente Ley de Transparencia española de 2013, no está muy bien posicionada en comparación con leyes promulgadas en periodos similares. Nuestro país aparece en el lugar 70, dentro una la lista de un total de 102 leyes analizadas, con 73 puntos de 150 , mientas que países como Croacia, República de Sierra Leona y República de Sudán del Sur aparecen en los primeros lugares, con valores que alcanzan 126, 122 y 120 puntos, respectivamente.

Para realizar un análisis más detallado, se ha relacionado este ranking con el Corruption Perceptions Index (2015). Este Índice de la Percepción sobre Corrupción (IPC) consiste en un estudio elaborado por la organización Transparency International que mide la ausencia o presencia de percepción de la corrupción, mediante una escala de 0 a100, donde 0 corresponde a un país interpretado como corrupto y 100 como muy transparente.

Este índice, que compara 168 países en el año 2015, muestra las siguientes valoraciones obtenidas por encima de 80 puntos: Dinamarca (91), Finlandia (90), Suecia (89), Nueva Zelanda (88), Holanda (87), Noruega (87), Suiza (86), Singapur (85), Canadá (83), Alemania (81), Luxemburgo (81) y Reino Unido (81), por lo que aparecen con un bajo nivel de percepción de la corrupción. Aunque el Índice de la Percepción sobre Corrupción no constituya un dictamen concluyente sobre los niveles de corrupción de las naciones, el estudio ubica a España en una posición intermedia del IPC con 58 puntos (Gráfico 1).

Este nivel de percepción de la corrupción viene avalado también por los datos del barómetro del Centro de Investigaciones Sociológicas (CIS), publicados en febrero de 2016 , donde el $47,5 \%$ de los españoles considera la corrupción como el segundo mayor problema de España, solo superado por el paro. Los datos muestran que la preocupación por la corrupción y el fraude ha crecido casi ocho puntos con respecto a la encuesta de enero (del $39,2 \%$ al $47,5 \%$ ), mientras que la del paro se ha mantenido casi estable.

Una vez analizado el Corruption Perceptions Index (IPC) (2015), que mide los niveles percibidos de corrupción en el sector público, así como el
Global Right to Information Rating (RTI Rating), que evalúa la calidad de las leyes de acceso a la información, se comparan los datos de ambos estudios (Gráfico 1).

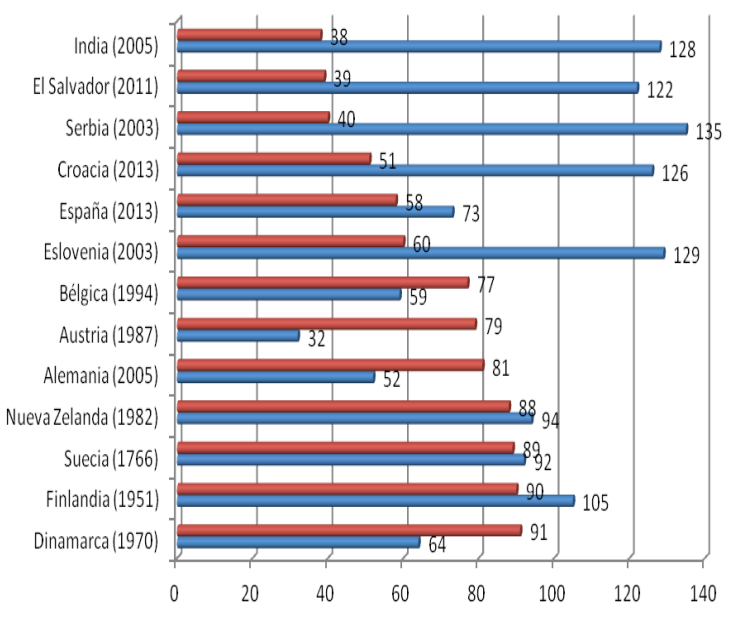

Corruption Perceptions Index 2015 (0 : se le percibe como sumamente corrupto y 100: se le aprecia como muy transparente)

- Global Right to Information Rating (0: valor minimo y 150: valor máximo)

Gráfico 1. Índice de percepción de la corrupción y calidad de las leyes de acceso a la información (Elaboración propia a partir de IPC y RTI Rating)

Dinamarca, que ocupa la primera posición como el país con menor índice de percepción de la corrupción (91 de 100), aparece, sin embargo, con un total de 64 puntos de 150, en la evaluación de su legislación de acceso a la información, con una ley del año 1970. Esta relación demuestra que la transparencia tiene más que ver con los valores éticos y la cultura intrínseca en la sociedad que con los mecanismos habilitadores de la misma (Gráfico 1).

Asimismo, se observa cómo, en general, los países con una mejor legislación tienen mayor percepción de corrupción. Por ejemplo, Liberia, India y El Salvador están entre los diez países con mejores leyes de transparencia y con mayor percepción de la corrupción (Gráfico 1).

Por otra parte, España muestra una posición intermedia en el Índice de Percepción de la Corrupción (posición 58 de 100), con una Ley de transparencia que nos sitúa en el extremo inferior (73 de 150). Si comparamos estos datos, por ejemplo, con Croacia, cuya promulgación de ley coincide en el mismo período (2013), observamos que este país con un alto Índice de Percepción de la Corrupción (51 de 100), se sitúa en extremo superior del ranking que analiza la calidad de las leyes de acceso a la información (126 de un máximo de 150 puntos) (Gráfico 1). 
Siguiendo los datos de ambos estudios, se observa que el desarrollo de una legislación sobre transparencia no impide que exista todavía un alto nivel de percepción sobre aspectos como la corrupción. Se concluye que en aspectos de transparencia influye más la tradición política y administrativa del país que el nivel de regulación al respecto.

\section{La transparencia en la política española}

El concepto de transparencia empieza a estar presente en el debate político español a raíz de la crisis. En este contexto, gobierno e instituciones perciben la deslegitimación que sufren en su actuación y la necesidad de superarla. Entre las respuestas a esta crisis de legitimidad, una de las más importantes es el desarrollo de una política de transparencia.

La transparencia aparece actualmente en el ideario de todos los partidos políticos, vinculada al concepto de regeneración democrática: recuperar el sentido de la utilidad política y transparencia aplicada al propio partido, con códigos éticos para sus miembros.

EI PSOE es uno de los partidos políticos que ha desarrollado su portal de transparencia, con información económica sobre el propio partido, información política y organizativa (actividad parlamentaria, viajes de los parlamentarios) y un buscador para acceder a los perfiles de cargos y candidatos. Asimismo, incluye un código ético, de obligado cumplimiento para todos los que están en un cargo público u orgánico.

Por lo que respecta al PP, la transparencia aparece en su portal ligado al concepto de regeneración. En su Plan de Regeneración Democrática incluye 70 medidas contra la corrupción que se articulan en cuatro ejes: $1^{\circ}$ ) Ley de transparencia, acceso a la información pública y buen gobierno; $2^{\circ}$ ) Medidas penales y procesales de lucha contra la corrupción/ Reforma del Código Penal; $3^{\circ}$ ) Ley Reguladora del Estatuto del Alto Cargo; y $4^{\circ}$ ) Ley de actividad económica de los partidos políticos. Aunque incluye un apartado dedicado a un código de buenas prácticas, solo dispone de las cuentas anuales del Partido Popular a partir del 2014.

En cuanto a los partidos emergentes (Ciudadanos y Podemos), la transparencia ocupa también un lugar destacado, siendo Ciudadanos el primer partido en el que la transparencia fue contemplada como una medida de regeneración democrática. En este sentido, su proyecto contempla propuestas importantes: ${ }^{a}$ ) Reforma de los par- tidos políticos, y $2^{\mathrm{a}}$ ) Independencia del Poder Judicial y de los Organismos Reguladores y Supervisores.

Del mismo modo, Podemos materializa en su portal de transparencia muchos de los postulados recogidos en las plataformas ciudadanas y en el $15 \mathrm{M}$, que se concretan en tres ejes: $1^{\circ}$ ) Cuentas claras, cargos públicos y cargos internos; $2^{\circ}$ ) La financiación del partido queda regulada en el Procedimiento de Control interno; y $3^{\circ}$ ) Cargos públicos e internos, que ofrece información sobre Actividad, Funciones, Salario, Declaración de bienes y Agenda).

\section{Metodología}

La apropiación de las redes sociales por parte de las administraciones ha provocado la aparición de espacios de comunicación con la ciudadanía. Así, los representantes políticos y sus partidos han iniciado en las redes un camino alternativo a la comunicación tradicional, que en la actualidad no puede disociarse de la actividad política.

La presencia de partidos políticos en las redes y su medición ha sido objeto de estudio de múltiples disciplinas como la Documentación y la Comunicación (Medina y Cañavate, 2014), la Sociología (Anduiza et al., 2010) y el propio Derecho (Gayán, 2014).

La definición de indicadores cuantitativos y cualitativos de la información en redes sociales es frecuente en la literatura sobre el tema. Así, este artículo se fundamenta especialmente en las aportaciones que miden la actividad parlamentaria y política en las redes sociales. En este sentido, a nivel nacional, cabe destacar los estudios de Araújo y González (2011) y Túñez y Sixto (2011). A nivel internacional autores como Cogburn y Espinoza-Vasquez (2011) ponen el foco en la campaña de 2008 de Barack Obama, hito, sin duda, en interacción político-ciudadano. Los indicadores y herramientas de medición aplicadas (Tweets, Klout), se ponen también de manifiesto en el más reciente artículo de Martínez-Rolán y Piñeiro-Otero (2014).

Para abordar este estudio se ha planteado una metodología en dos fases sucesivas: primeramente, se han medido las diferentes dimensiones de la participación en Twitter de los cuatro principales partidos políticos españoles: Partido Popular, Partido Socialista Obrero Español, Podemos y Ciudadanos. Para ello, se escogen y formulan métricas (KPI) frecuentes en este tipo de estudios, dedicadas a la medición de aspectos como "popularidad", "actividad" o "influencia" Con estos datos se pretende ofrecer una visión global 
del grado de implantación y participación de estas instituciones en la creación de espacios conversacionales.

Una vez analizados estos datos, en una segunda fase, se realiza un análisis sobre el contenido de los mensajes sobre Transparencia en la red Twitter, por ser la más habitual y dinámica. Para ello, se ha propuesto una categorización que permite tipificar la orientación de la Transparencia en dos ámbitos:

1. El uso del término en el discurso político en Twitter:

- Transparencia incluida como hashtag, tomada como herramienta principal para detectar el eco del concepto en las conversaciones políticas.

- Transparencia incluida como término en el discurso, que nos permitirá evaluar el nivel de uso del concepto en estas plataformas.

2. Análisis contextual del uso del término "Transparencia":

- Transparencia relacionada con la actividad interna de los partidos.

- Transparencia relacionada con la corrupción y petición de transparencia a otros partidos.

La recogida de estos datos se llevó a cabo manualmente y a través de herramientas de métrica social, disponibles en el mercado como Top Influencers para hallar el Klout. Para el análisis de contenido se utilizó la búsqueda avanzada de Twitter, que permite localizar Hashtags y texto libre y filtrarlos por rango de fechas y por autoría (@ppopular, @Psoe, @ahorapodemos, @CiudadanosCs). Se decidió excluir los retweets de la búsqueda, centrándonos únicamente en los mensajes originarios de las plataformas de los partidos.

El estudio se realizó en dos períodos:

- Preelectoral (septiembre de 2015) cuándo se estaban configurando los programas políticas. Los resultados de este estudio se presentaron en el monográfico sobre Transparencia y Buen Gobierno del VIII Coloquio sobre Brecha Digital e Inclusión Social, celebrado en la Universidad Carlos III de Madrid en 2015.

- Postelectoral (desde el 20 de diciembre de 2015, fecha de las elecciones generales hasta abril de 2016) para verificar la aplicabilidad y profusión del término en una etapa compleja, marcada por las negociaciones y pactos entre partidos.

\section{Resultados de las métricas en Twitter de los partidos políticos}

Para contextualizar la actividad en Twitter se han medido las siguientes categorías: publicaciones (tweets), seguidores y seguidos (Gráfico 2).

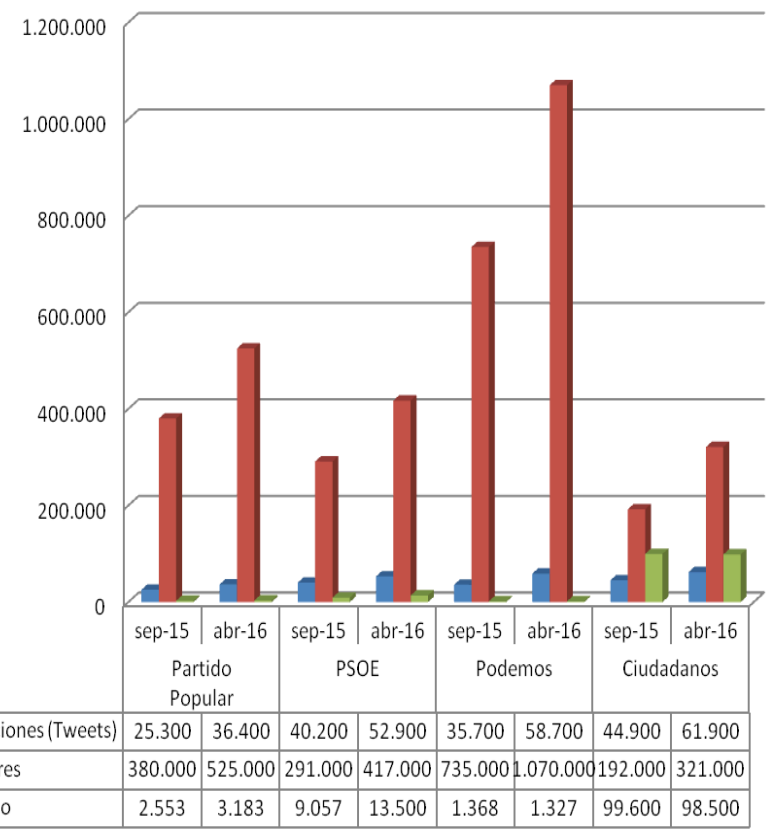

Gráfico 2. Análisis métrico de Twitter

Ciudadanos es el perfil más antiguo en esta red y también el que más contenido ha generado hasta la fecha. Creado en enero de 2009, contaba en abril de 2016 con 61.900 publicaciones, con un incremento de $27,4 \%$ si lo comparamos con el primer estudio de septiembre (2015) (Gráfico 2).

En cuanto a seguidores, Podemos, con un perfil más joven (enero de 2014), es el partido con más seguidores (1.070.000) (Gráfico 2). Lo cual da idea de su poder de captación hacia un público más joven o más vinculado a las TIC como mecanismo de expresión.

Por lo que respecta a la influencia, se ha escogido el índice Klout (1), que mide entre en 0 y 100 diferentes dimensiones del impacto de una cuenta de Twitter. El mayor impacto en cuanto a influencia lo presenta el perfil del PSOE, con un Klout de 87. Sin embargo, pese a que Ciudadanos genera más publicaciones y Podemos tiene más seguidores, el Klout de ambos partidos no es tan elevado, ocupando respectivamente el segundo y tercer puesto con 80 y 79 (Tabla I). En este sentido, es importante destacar el valor de aspectos como la interacción o el compromiso frente al número de seguidores o publicaciones. 


\begin{tabular}{lcc}
\hline & Septiembre 2015 & Abril de 2016 \\
\hline PSOE & 86 & 87 \\
\hline Ciudadanos & 83 & 80 \\
\hline Podemos & 78 & 79 \\
\hline Partido Popular & 78 & 78
\end{tabular}

Tabla I. índice Klout a partir de topinfluencers.net

\section{Resultados del Análisis de Contenido: uso y análisis contextual del término 'Transparencia'}

El total de tweets contabilizados relacionados con la Ttansparencia en todo el período del análisis es de 551 , de los cuales casi un $85 \%$ corresponden a los partidos emergentes $(48,73 \%$ a Ciudadanos y $36,01 \%$ a Podemos).

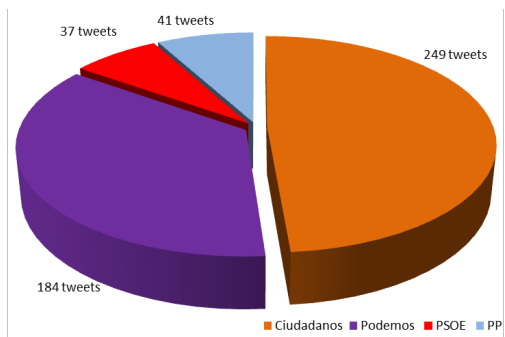

Gráfico 3. Número de Tweets relacionados con la transparencia

Tipificando los resultados obtenidos, se establecieron las conclusiones en función de dos categorías.

En primer lugar, se analizó el uso del término 'transparencia' en el discurso político en Twitter, en particular del hashtag \#transparencia y del uso del término en el discurso (Gráfico 4).

Los resultados muestran que el uso del término "Transparencia" como hashtag, o incluso como término, no es frecuente en ninguno de los dos periodos analizados. De un total de 55 tweets contabilizados en ambas etapas, 34 pertenecen a Ciudadanos $(61,8 \%)$, mientras que el $38,2 \%$ se reparte entre el resto de los partidos: Podemos $(18,18 \%)$, PSOE $(18,18 \%)$ y PP $(1,81 \%)$.

En segundo lugar se realizó un nálisis contextual del término 'transparencia' (Gráfico 5), pues el hecho de que no se emplee el término transparencia, no implica que no esté implícito en el discurso. El análisis contextual del uso del término se basó en el establecimiento de dos categorías: transparencia relacionada con la actividad interna de los partidos, y transparencia relacionada con la corrupción o petición de transparencia a otros partidos.

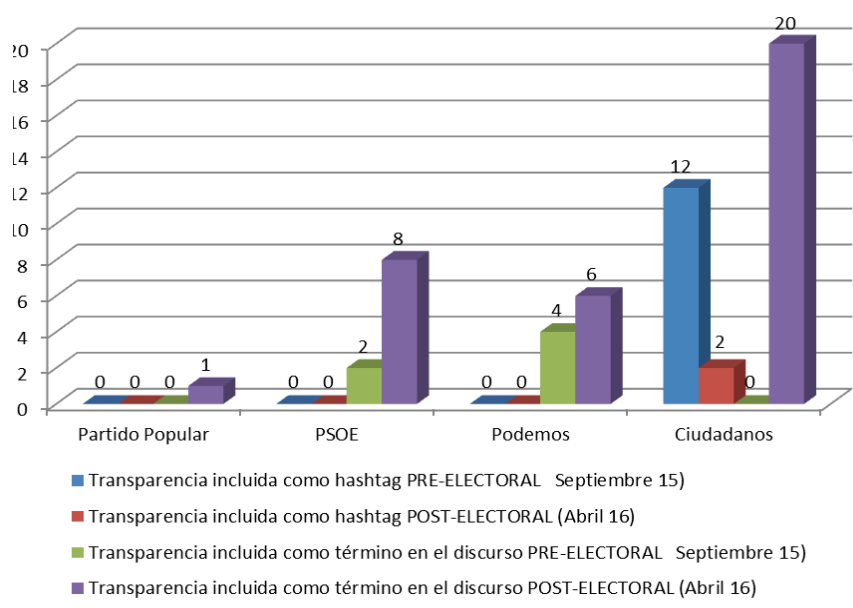

Gráfico 4. Tweets relacionados con el término $y$ el hashtag transparencia

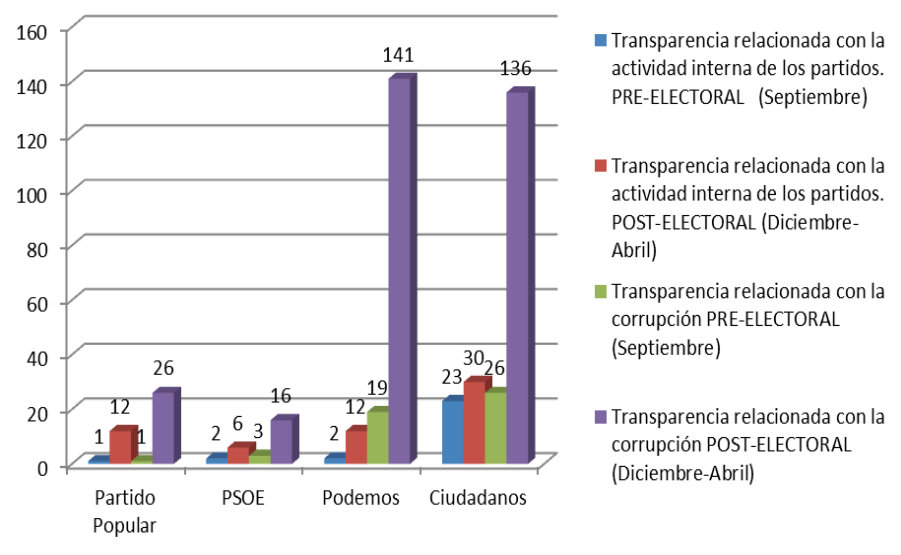

Gráfico 5. Análisis contextual de Tweets

En cuanto a la transparencia relacionada con la actividad interna de los partidos, el análisis muestra que de un total de 88 tweets, Ciudadanos vuelve a liderar esta categoría con 53 tweets, lo que constituye un $60 \%$ si contabilizamos ambos periodos.

Por otra parte en la categoría de transparencia relacionada con la corrupción o petición de transparencia a otros partidos, nuevamente, las formaciones emergentes ocupan las primeras posiciones (Ciudadanos con 162 tweets, seguido de Podemos con 160 tweets de un total de 368).

A tenor del estudio realizado, se observa que, si bien el hashtag \#transparencia y el término no aparecen con una presencia significativa, sí es relevante su presencia dentro del contexto donde se debaten conceptos vinculados a este tema 
como la corrupción y la regeneración democrática.

Por otra parte, su articulación en las redes sociales ha sido distinta en ambos periodos. Si bien en el análisis de septiembre su presencia fue más moderada, en los meses posteriores si se nota un repunte en los discursos de todos los partidos políticos.

\section{Conclusiones}

La sola promulgación y estructura de la ley no implica una mayor transparencia. En muchos casos la demanda o necesidad de una norma indica la situación problemática de las cuestiones a las que hace referencia.

El nuevo sistema de gestión política exige la participación ciudadana. Las redes sociales constituyen una plataforma excepcional para contactar y difundir el mensaje político de manera más inmediata.

Sin embargo, la presencia de los partidos en las redes puede quedarse en un aspecto meramente formal si no se aplican medidas de alto calado para reformular y aceptar la participación ciudadana. Que un partido esté en las redes no implica que sea más abierto.

Lo mismo sucede con el análisis de aspectos cuantitativos. El hecho de destacar en seguidores o en publicaciones no lleva implícito llevar a cabo una política más efectiva en las redes sociales, basada en criterios de búsqueda de impacto y de fidelización.

Los partidos emergentes, fruto en parte del descontento ciudadano con los cauces de la política tradicional, son más activos a la hora de recalcar en sus mensajes su transparencia interna o demandarla a otros partidos.

Al finalizar este estudio, se propone continuar esta línea de investigación contemplando nuevas categorías de evaluación como los retweets o los perfiles de los líderes y de los partidos autonómicos y locales, lo que permitiría obtener una perspectiva más global.

\section{Notas}

(1) Servicio web que mide a través de un índice la influencia entre 0 y 100 de los usuarios de redes sociales basándose en aspectos como: seguidores, seguidos, contenido, retweets así como otros elementos menos transparentes para el usuario final. Para más información véase: Klout www.klout.com

\section{Referencias}

Anduiza, E.; Cantijoch, M.; Colombo, C.; Gallego, A.; Salcedo, J. (2010). Los usos políticos de Internet en España.
// Revista española de investigaciones Sociológicas. 129:1 (2010),133-146.

Araújo, J. R.; González, B. D. (2011). Los diputados del parlamento gallego en Facebook. // Redmarka: revista académica de marketing aplicado. 7:1 (2011), 77-106.

Centre for Law and Democracy. Global Right to Information Ranking. http://www.rti-rating.org/ (2016-04-01).

Centro de Investigaciones Sociológicas (CIS). // Barómetro febrero de 2016. http://www.cis.es/cis/opencms/ES/Noti ciasNovedades/InfoCIS/2016/Documentacion_3131.html (2016-04-01)

Ciudadanos. // Portal de transparencia. https://www.ciuda danos-cs.org/nuestro-proyecto (2016-04-01).

Cogburn, D. L.; Espinoza-Vasquez, F. K. (2011). From networked nominee to networked nation: Examining the impact of Web 2.0 and social media on political participation and civic engagement in the 2008 Obama campaign. // Journal of Political Marketing. 10:1-2 (2011), 189-213.

España. Ley 19/2013, de 9 de diciembre, de Transparencia, Acceso a la Información Pública y Buen Gobierno. BOE. núm. 295, de 10 de diciembre de 2013. https:// www.boe.es/buscar/act.php?id=BOE-A-2013-12887 (2016-04-01)

España. Ley 37/2007 sobre Reutilización de la Información del Sector Público. // BOE. 276 (17 de noviembre de 2007), 47160-47165. https://www.boe.es/diario_boe/txt.p hp?id=BOE-A-2007-19814 (2016-04-01).

Gastón, Concha; Naser, Alejandra. (2012). El desafío hacia el gobierno abierto en la hora de la igualdad. Santiago de Chile: Comisión Económica para América Latina y el Caribe (CEPAL). http://www.cepal.org/ddpe/publicacio nes/xml/9/46119/w465.pdf (2016-04-01).

Gayán, S. C. (2014). Marco normativo e institucional del nuevo derecho de participación y las TIC: análisis desde las experiencias autonómicas. // IDP. 19, 48-61.

Martínez-Rolán, X.; Piñeiro-Otero, T. (2014). Del perfil electoral a la interacción con la ciudadanía. La apropiación de Twitter por los agentes del Parlamento de Galicia. // Revista de la Asociación Española de Investigadores en Comunicación. 2014.

Medina, D. E. M.; Cañavate, A. M. (2014). La información parlamentaria en los parlamentos regionales en España: evaluación de las sedes web y presencia en los medios sociales. // Revista General de Información y Documentación. 24:1, 67-98.

Partido Popular. Portal de transparencia. http://www.pp.es/ transparencia (2016-04-01).

Podemos. Portal de transparencia. https://transparencia. podemos.info/ (2016-04-01).

Portal de transparencia. Gobierno de España. http://trans parencia.gob.es/ (2016-04-01).

PSOE. Portal de transparencia. http://www2.psoe.es/transpa rencia/ (2016-04-01).

Transparency International. Corruption Perceptions Index (2015). http://www.transparency.org/cpi2015 (2016-04-01).

Túñez, M.; Sixto, J. (2011). Redes sociales, política y Compromiso 2.0: La comunicación de los diputados españoles en Facebook. // Revista Latina de comunicación social. 66, 1-25.

VIII Coloquio sobre Brecha Digital e Inclusión Social (2015). Madrid: Universidad Carlos III de Madrid. http://portal. uc3m.es/portal/page/portal/inst_docum_gest_info_agustin_millares/VIII_Brecha_digital (2016-04-01).

Enviado: 2016-04-04. Segunda versión: 2016-09-14 Aceptado: 2016-09-22. 